\title{
Simulation of Natural Convection in a Cavity: Cooling with Top versus Bottom Partition
}

\author{
Emel Evren Selamet ${ }^{1}$ and Ahmet Selamet ${ }^{2}$ \\ 1. Mechanical and Aerospace Engineering, Center for Automotive Research, Ohio State University, Columbus, OH 43212, USA \\ 2. Mechanical and Aerospace Engineering, Ohio State University, Columbus, OH 43210, USA
}

\begin{abstract}
Natural convection in a square cavity is studied numerically with vertical hot walls and a cold partition located at the bottom or top walls. The effect of orientation of partition on isotherms, flow patterns, and heat transfer is analyzed for different fluids and Rayleigh numbers. The placement of partition is found to considerably influence the isotherms, flow patterns, and heat transfer. Heat transfer rate is observed always to increase when the partition is moved from bottom to top wall.
\end{abstract}

Key words: Natural convection, Rayleigh number, Prandtl number.

\section{Introduction}

Natural convection in differentially heated cavities has been investigated extensively both experimentally and computationally due to its importance in many applications, including, for example, cooling of electronic components, heat exchangers in industrial processes, and energy-efficient buildings. Several researchers focused specifically on a partition in the cavity and analyzed its impact in terms of enhancement/suppression of heat transfer. Majority considered cavities with a horizontal temperature gradient and conducting or nonconducting partitions. An early experimental work on natural convection in an enclosed vertical fluid layer with a vertical baffle was conducted by Emery [1] to model a coolant channel in a nuclear reactor. This study observed a fairly significant change in local temperature profiles due to the vertical baffle though not appreciable reduction in heat transfer. Among other experimental efforts, Nansteel and Greif [2, 3] investigated natural convection in a cavity with vertical hot and cold walls, insulated horizontal walls, and a thin nonconducting partition on the ceiling. These experimental studies

Corresponding author: Emel Evren Selamet, Ph.D., research scientist, research field: thermal science. were carried out with water and for $\mathrm{Ra}>10^{9}$. Olson et al. [4] considered also high $\mathrm{Ra}$ in an enclosure with a partition extending from the floor in a building application. Oztop and Bilgen [5] investigated heat transfer in a cavity with a cooled partition and uniform heat generation. Anilkumar and Jilani [6] considered a nanofluid-filled cavity with a heated partition at the bottom insulated wall along with cooled side walls. Due to their steady state formulation, the computational domain in Ref. [6] was half of the enclosure.

The present numerical study examines the time-dependent natural convection in a cavity with hot side walls, insulated horizontal walls, and a cooled partition. The objective here is to examine the importance of location of cooled partition (bottom vs. top) on overall heat transfer with variety of working fluids.

\section{Physical Model}

Figs. $1 \mathrm{a}$ and $1 \mathrm{~b}$ show the schematic of the square enclosure considered here. This cavity has hot side walls maintained at $T_{\mathrm{H}}$, horizontal adiabatic walls, and a cold partition at $T_{\mathrm{C}}$ placed in the middle of either (a) bottom or (b) top insulated wall. Height and width of the partition is $1 / 2$ and $1 / 10$ of the length of surrounding walls, respectively. The primary goal of the study is to 


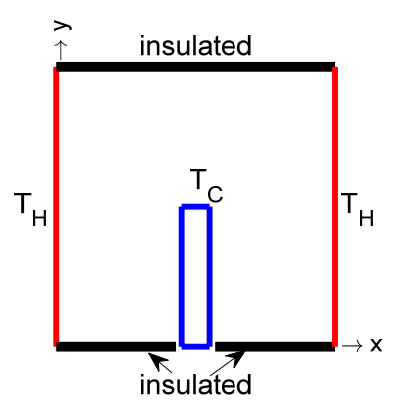

(a)

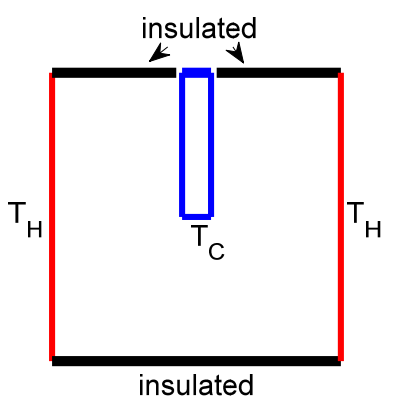

(b)

Fig. 1 Schematic of the enclosure with (a) bottom, (b) top partition.

investigate the natural convection heat transfer with these two different partition arrangements.

In terms of the following dimensionless variables $x$ $=x^{*} / L, y=y^{*} / L, u=u^{*} L / \alpha, v=v^{*} L / \alpha ; t=t^{*} \alpha / L^{2} ; p=$ $p^{*} L^{2} /\left(\rho \alpha^{2}\right) ; \theta=\left(T-T_{\mathrm{C}}\right) /\left(T_{\mathrm{H}^{-}} T_{\mathrm{C}}\right)$ where $x^{*}, y^{*}$ are the coordinates; $u^{*}, v^{*}$ the velocities; $t^{*}$ the time; $p^{*}$ the pressure; $T$ the temperature; $L$ the length of the enclosure wall, two dimensional governing equations for mass $\mathrm{x}$ - and $\mathrm{y}$-momentum and energy may be written for buoyancy-driven incompressible fluids as:

$$
\begin{gathered}
\frac{\partial u}{\partial x}+\frac{\partial v}{\partial y}=0, \\
\frac{\partial u}{\partial t}+\frac{\partial u^{2}}{\partial x}+\frac{\partial u v}{\partial y}=-\frac{\partial p}{\partial x}+\operatorname{Pr} \nabla^{2} u, \\
\frac{\partial v}{\partial t}+\frac{\partial u v}{\partial x}+\frac{\partial v^{2}}{\partial y}=-\frac{\partial p}{\partial y}+\operatorname{Pr} \nabla^{2} v+\operatorname{Ra} \operatorname{Pr} \Theta, \\
\frac{\partial \Theta}{\partial t}+\frac{\partial u \Theta}{\partial x}+\frac{\partial v \Theta}{\partial y}=\nabla^{2} \Theta,
\end{gathered}
$$

where $\nabla^{2}$ is the two-dimensional Laplacian; $\operatorname{Pr}=v / \alpha$, the Prandtl number; $\mathrm{Ra}=g \beta \Delta T L^{3} / v \alpha$, the Rayleigh number which accounts for the coupling between energy and momentum balances, $g$ the gravitational acceleration, $\beta$ the coefficient of thermal expansion of the fluid, $\Delta T$ the temperature difference between hot and cold boundaries; $v$ the kinematic viscosity; and $\alpha$ the thermal diffusivity. Boussinesq approximation, which treats density as a constant in all equations except for the gravitational force term in the momentum equation, is used. Even though the computations are performed by using primitive variables, flow field results are presented in terms of the stream function $\Psi(u=\partial \Psi / \partial y, v=-\partial \Psi / \partial x)$. No slip boundary conditions are imposed on all walls. Unsteady governing equations are employed here since the evolution of flow pattern and the associated temporal behavior can only be captured by using time-dependent computations. The governing equations with primitive variables are solved on a staggered grid by a numerical scheme developed by the lead author applying the Godunov scheme to convective terms and centered finite difference to diffusive terms. Details of the method employed here have been described in an earlier work [7]. The current computational results are presented in the next section.

\section{Results}

The accuracy of the numerical approach employed in the present study has already been validated through earlier comparisons [7] with the benchmark numerical solution of Davis [8], therefore such details are avoided here. In addition, a specific comparison is included here with Ref. [2], which is relevant to the present study. Nansteel and Greif [2] investigated the natural convection experimentally in an enclosure with an aspect ratio of $1 / 2$ and a vertical partition for $\mathrm{Ra}$, based on the height of cavity, $1.25 \times 10^{9}-1.375 \times 10^{10}$. They indicated that temperature profiles were virtually independent of $\mathrm{Ra}$ over the chosen range. Present computations are carried out only for $\mathrm{Ra}=10^{8}$ to avoid too small of a grid size and time step of higher Ra. The predictions here for vertical temperature variation at 


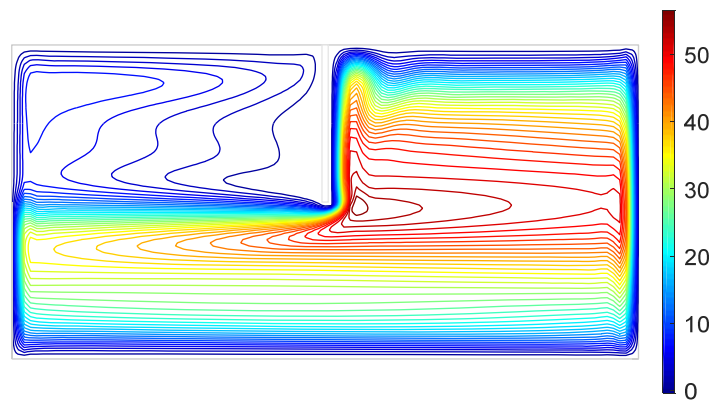

Fig. $2 \operatorname{Pr}=3.5:$ Streamlines for $\mathrm{Ra}=10^{8}$.

locations of $1 / 4$ and $3 / 4$ of the cavity length exhibit a trend qualitatively similar to those measured by Nansteel and Greif [2] despite the difference in Ra considered for comparison. Streamlines shown in Fig. 2 at $\mathrm{Ra}=10^{8}$ from the current numerical simulations are consistent with flow patterns of Nansteel and Greif [2]. Similar to Nansteel and Greif [2], main flow does not penetrate much into the upper left quadrant of the enclosure and flow separation occurs behind the partition.

The results of the square cavity described in Fig. 1 will be presented next in terms of streamlines and temperature profiles (isotherms), as well as the rate of heat transfer from the surfaces for $\operatorname{Pr}=0.024$ (liquid metal), 0.71 (air), 6 (water), and 450 (silicon oil) at $\mathrm{Ra}$ $=10^{4}-10^{6}$. Numerical study is performed with $100 \times$ 100 number of nodes after its adequacy is tested. Heat transfer rate is characterized by Nusselt $(\mathrm{Nu})$ number which is the dimensionless temperature gradient integrated over the wall.

Fig. 3 shows (a) streamlines and (b) isotherms for $\operatorname{Pr}=0.024$ with both BP (bottom partition) and TP (top partition). Two steady cells rotating in the opposite direction appear at $\mathrm{Ra}=10^{4}$. The flow rate reaches maximum at the center of circulations. Temperature gradually drops from hot walls to the cold partition, as expected. For higher Ra, unsteady solution is obtained resulting in multiple cell structures. As an example, streamlines and isotherms are shown at a specific time for $\mathrm{Ra}=5 \times 10^{5}$. Isotherms are now more distorted as a result of the stronger fluid motion in the enclosure particularly for the TP case. Because of the unsteady oscillatory nature of convection at low $\operatorname{Pr}$ fluids, flow and temperature fields are no longer symmetric around the partition.

Fig. 4 shows (a) streamlines and (b) isotherms for $\operatorname{Pr}=0.71$ at various Ra. Again, two counter-rotating zones are present at $\mathrm{Ra}=10^{4}$. For higher $\mathrm{Ra}$, the center of rotating zones is elongated creating two inner smaller circulations for $\mathrm{BP}-\mathrm{a}$ behavior not observed for TP. Higher temperatures are prevalent right above the partition for $\mathrm{BP}$, creating a weak motion in contrast to the more vigorous one of TP. Flow patterns and isotherms exhibit steady symmetrical behavior around the partition for all the Ra numbers considered here. An example comparison is also shown in Fig. 5 in terms of vertical temperature profiles for BP vs. TP at $x=1 / 4$ and $\mathrm{Ra}=10^{5}$ for (a) $\operatorname{Pr}=0.024$ and (b) $\operatorname{Pr}=0.71$. These profiles for $\operatorname{Pr}=$ 0.024 and $\mathrm{Pr}=0.71$ are similar for BP (blue) unlike TP (brown). For $\operatorname{Pr}=0.024$ and TP, temperature first decreases until mid-y and then increases because of the multiple cell structure, whereas temperature of TP exhibits a nearly linear variation for $\operatorname{Pr}=0.71$.

Increasing Pr to 6 resulted in similar behavior of flow and temperature fields as for $\operatorname{Pr}=0.71$.

For $\operatorname{Pr}=450$ (Fig. 6), the flow field is similar to that of $\operatorname{Pr}=0.71$ and 6 with the exception of small top corner counter-rotating vortices, which alter the temperature fields as observed in Fig. 6b. Those weak top corner vortices get stronger with increasing $\mathrm{Ra}$ for $\mathrm{BP}$, whereas they remain weak for TP. Because of the formation of corner circulations in BP, the two main cells are somewhat pushed down which gives them a slightly different appearance than those of $\operatorname{Pr}=0.71$ and 6. Flow patterns and isotherms again exhibit steady symmetrical behavior around the partition for all the Rayleigh numbers considered here.

$\mathrm{Nu}$ on the left hot wall vs. Ra is depicted in Fig. 7 for different Pr where solid lines are for BP and dashed lines for TP. Comparison of the results reveals that heat transfer rate is increased noticeably with $\mathrm{TP}$ 
(a)
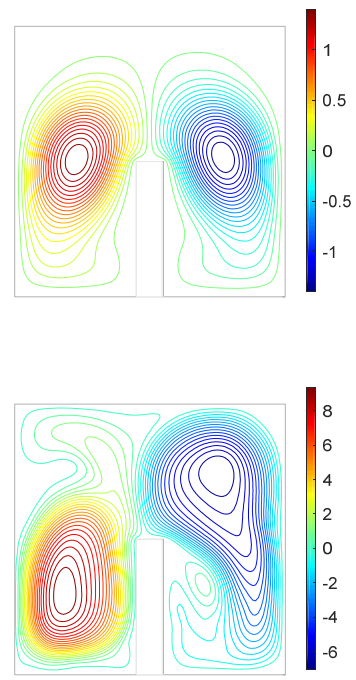

BP

(b)

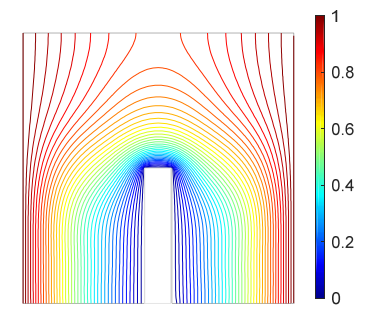

(a)

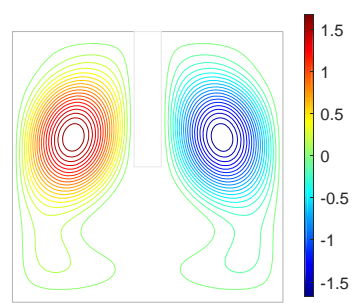

(b)
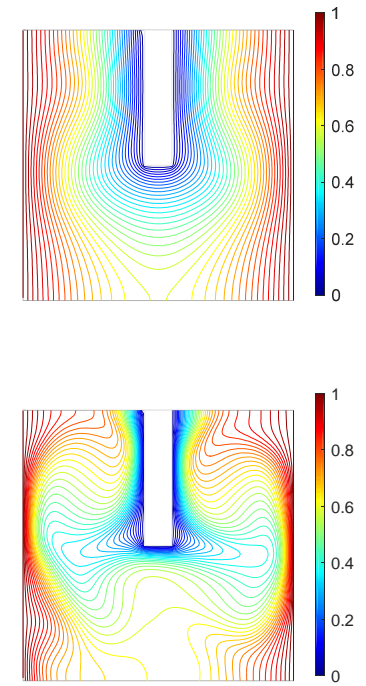

Fig. $3 \mathrm{Pr}=$ 0.024: (a) Streamlines and (b) isotherms for $\mathrm{Ra}=10^{4}$ and $5 \times 10^{5}$.

BP

(a)

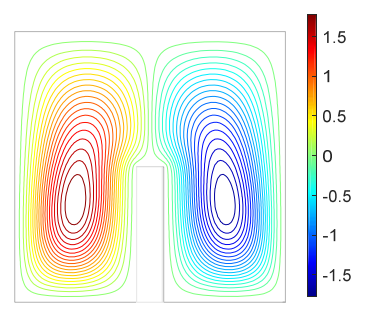

(b)

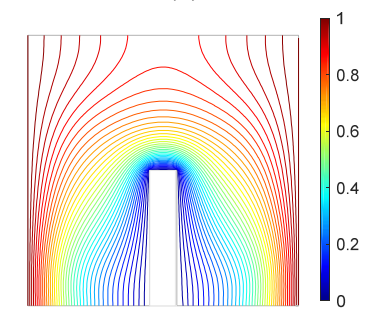

$\mathrm{Ra}=5 \times 10^{5}$
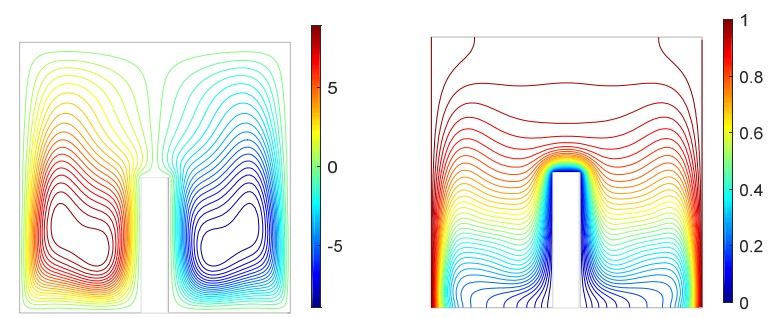

(a)

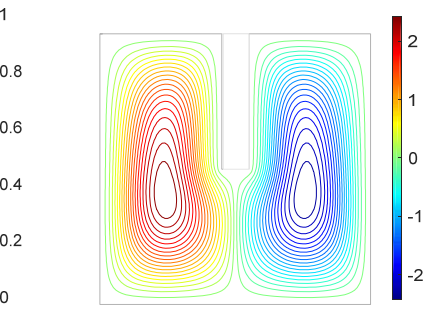

$$
\mathrm{Ra}=10^{4}
$$

TP

(b)

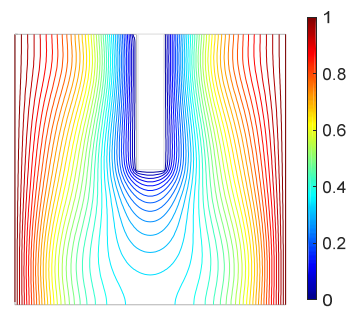

$$
\mathrm{Ra}=10^{6}
$$
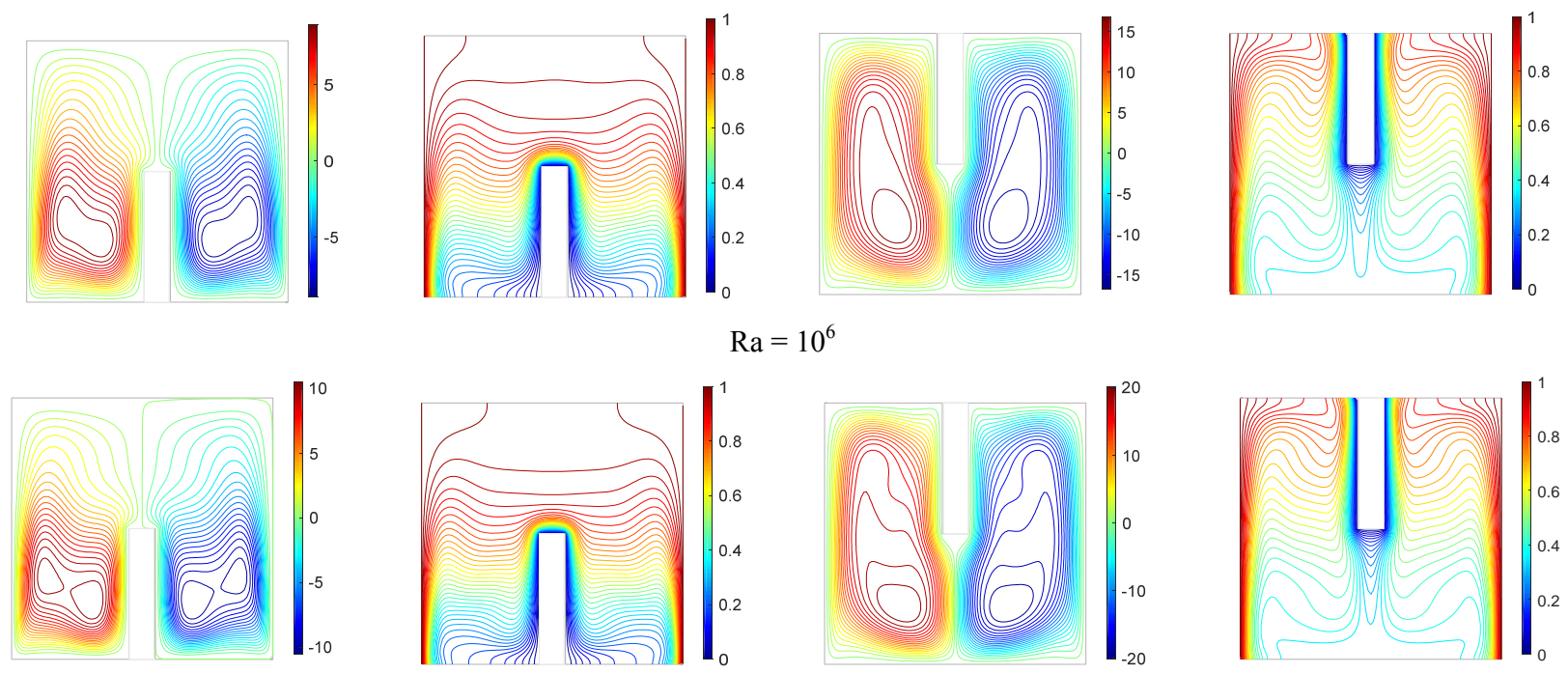

Fig. $4 \operatorname{Pr}=0.71$ : (a) Streamlines and (b) isotherms for $\mathrm{Ra}=10^{4}, 5 \times 10^{5}$, and $10^{6}$. 
$\operatorname{Pr}=0.024$

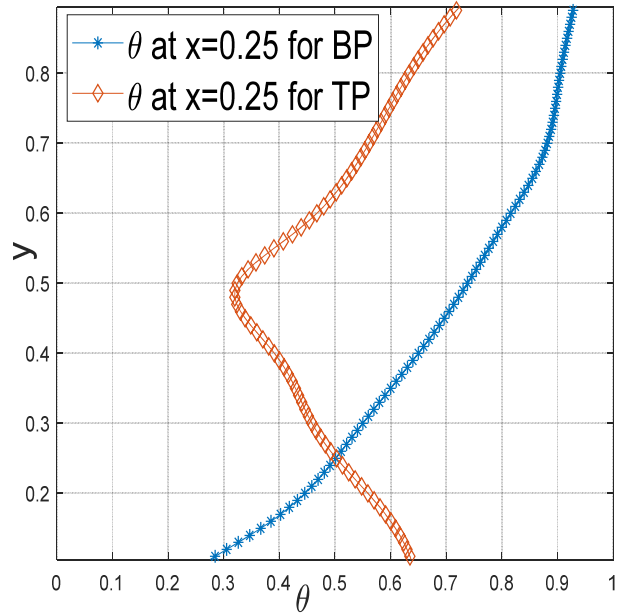

$\operatorname{Pr}=0.71$

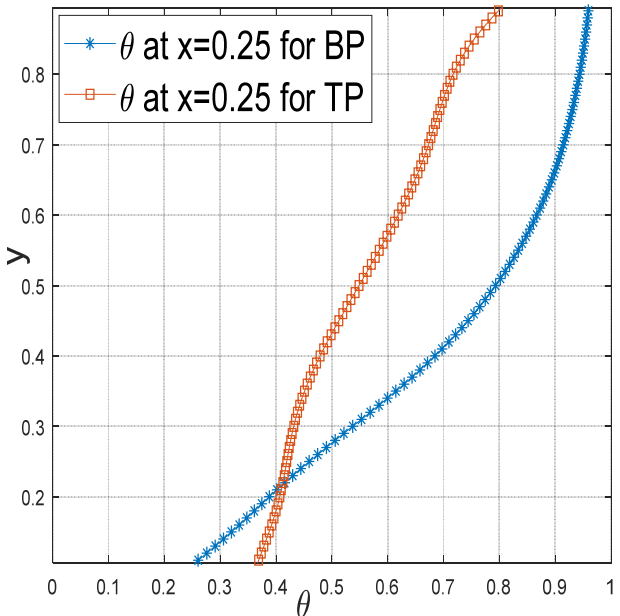

Fig. 5 Vertical temperature profiles for BP and TP cases at $x=1 / 4$ and Ra $=5 \times 10^{5}$.

BP

(a)
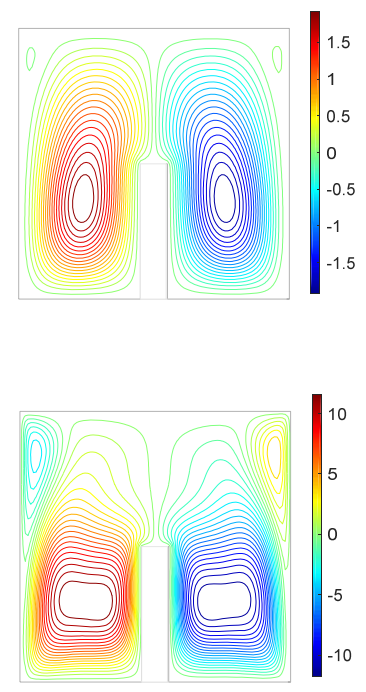

(b)

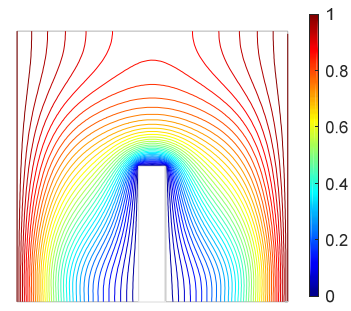

$\mathrm{Ra}=10^{4}$

$\mathrm{Ra}=5 \times 10^{5}$
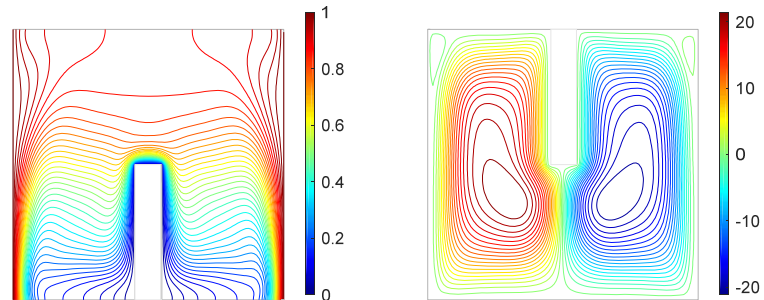

$\mathrm{Ra}=10^{6}$
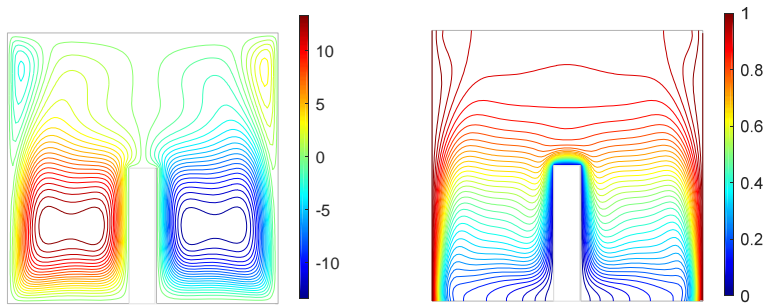

(a)

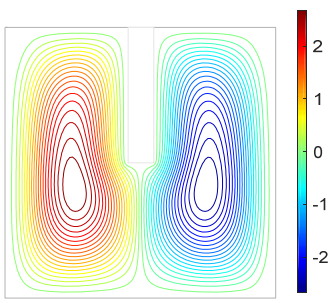

TP

(b)
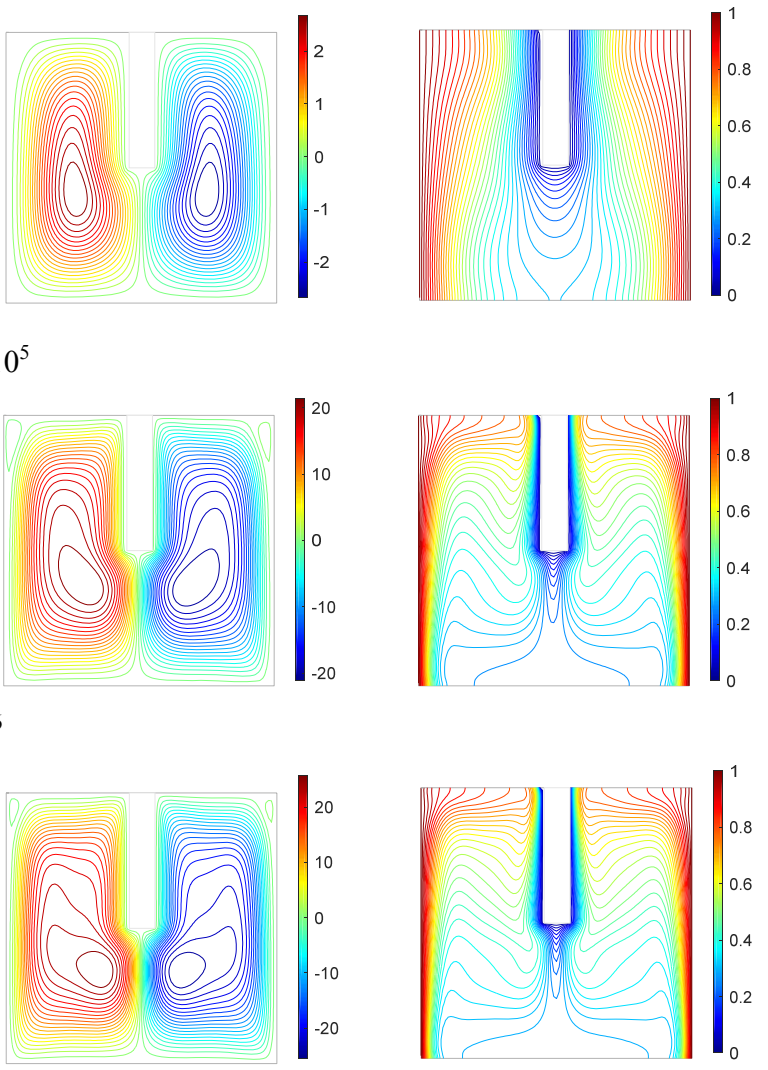

Fig. 6 Pr = 450: (a) Streamlines and (b) isotherms for $R a=10^{4}, 5 \times 10^{5}$, and $10^{6}$. 

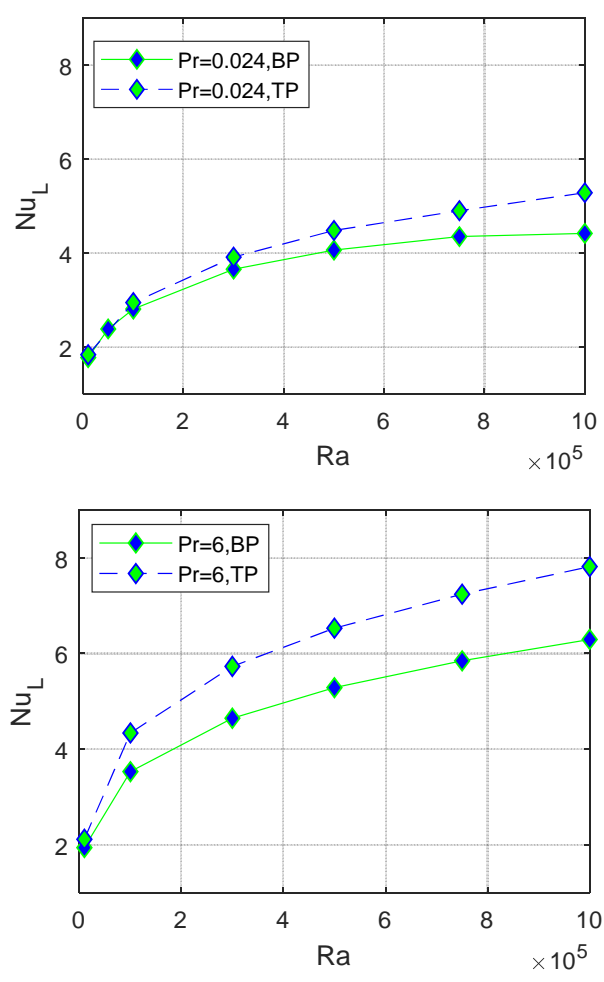
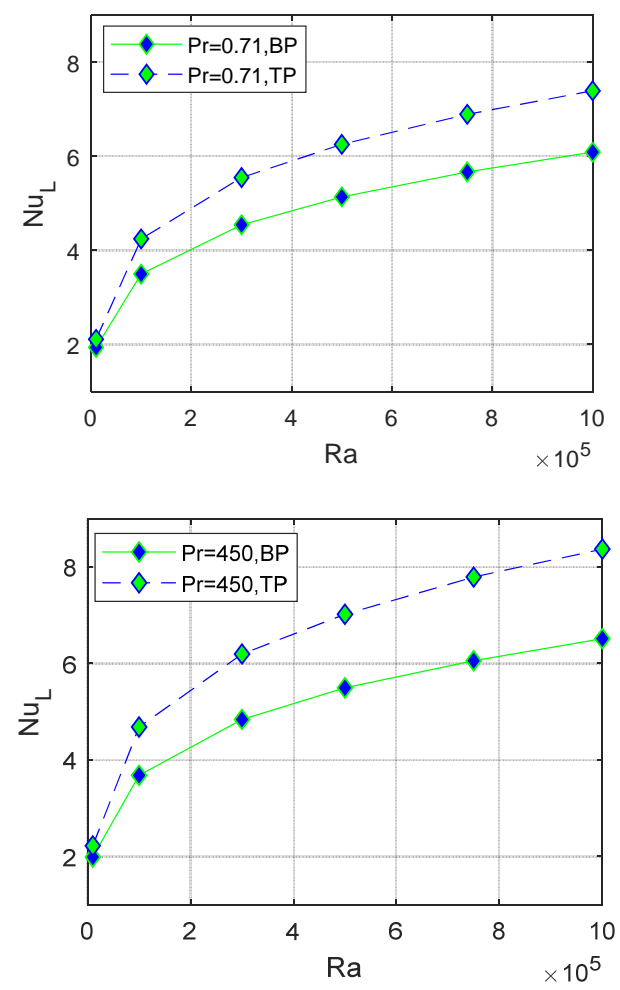

Fig. 7 Comparison of BP and TP in terms of Nu versus Ra for different working fluids.

relative to the $\mathrm{BP}$. For $\operatorname{Pr}=0.71,6$, and 450, steady solution is obtained for all Rayleigh numbers considered here, whereas the solution is unsteady for $\operatorname{Pr}=0.024$ when $\operatorname{Ra}>10^{4}$.

\section{Conclusion}

Time-dependent natural convection is studied numerically in a square cavity with hot side walls, insulated horizontal walls, and a cooled partition. The results reveal that the location (bottom vs. top) of the cooled partition, is considerably influential on heat transfer for all working fluids of $0.024<\operatorname{Pr}<450$. Placing the partition on top wall (rather than the bottom) increases the heat transfer rate for all Rayleigh numbers. This augmentation is more visible with increasing Ra. Finally, the enhancement of heat transfer is the least for $\operatorname{Pr}=0.024$ in contrast to higher Pr.

\section{References}

[1] Emery, A. F. 1969. "Exploratory Studies of Free-Convection Heat Transfer through an Enclosed Vertical Liquid Layer with a Vertical Baffle." Journal of
Heat Transfer 91: 163-5.

[2] Nansteel, M. W., and Greif, R. 1981. "Natural Convection in Undivided and Partially Divided Rectangular Enclosures.” Journal of Heat Transfer 103: 623-9.

[3] Nansteel, M. W., and Greif, R. 1984. "An Investigation of Natural Convection in Enclosures with Two- and Three-Dimensional Partitions." International Journal of Heat and Mass Transfer 27 (4): 561-71.

[4] Olson, D. A., Glicksman, L. R., and Ferm, H. M. 1990. "Steady-State Natural Convection in Empty and Partitioned Enclosures at High Raylleigh Numbers." Transactions of the ASME 112: 640-7.

[5] Oztop, H., and Bilgen, E. 2006. "Natural Convection in Differentially Heated and Partilaay Divided Square Cavities with Internal Heat Generation." International Journal of Heat and Fluid Flow 27: 466-75.

[6] Anilkumar, S. H., and Jilani, G. 2008. "Natural Convection Heat Transfer Enhancement in a Closed Cavity with Partition Utilizing Nano Fluids." In Proceedings of the World Congress on Engineering 2008, vol. II, pp. 978-88.

[7] Evren-Selamet, E., Arpaci, V. S., and Borgnakke, C. 1992. "Simulation of Laminar Buoyancy-Driven Flows in an Enclosure." Numerical Heat Transfer Part A 22: 401-20.

[8] Davis, G. V. 1983. "Natural Convection of Air in a Square Cavity: A Bench Mark Numerical Soluton.” International Journal of Numerical Methods in Fluids 3 (3): 249-64. 